\title{
Risk Factors of Influenza-Associated Necrotizing Encephalopathy in Children
}

Liu G', Yang S ${ }^{2}$, Li S ${ }^{1}$, Chen $Q^{1}$, Xiao $W^{3}$ and Li $P^{1 *}$ ${ }^{1}$ Department of Pediatric Emergency, Guangzhou Women and Children's Medical Center, Guangzhou Medical University, Guangzhou, Guangdong, P.R. China ${ }^{2}$ Department of Pediatric Neurology, Guangzhou Women and Children's Medical Center, Guangzhou Medical University, Guangzhou, Guangdong, P.R. China ${ }^{3}$ Department of Pediatric Radiology, Guangzhou Women and Children's Medical Center, Guangzhou Medical University, Guangzhou, Guangdong, P.R. China

*Corresponding author: Peiqing Li, Department of Pediatric Emergency, Guangzhou Women and Children's Medical Center, Guangzhou Medical University,

Guangzhou, Guangdong, P.R. China

Received: June 11, 2021; Accepted: July 06, 2021; Published: July 13, 2021

\section{Abstract}

Objective: A case-control study of Influenza-Associated Necrotizing Encephalopathy (IANE) in children was conducted to explore the risk factors for the diagnosis of IANE, and to provide a predictive reference for the diagnosis of IANE.

Methods: The children with IANE who received treatment in our hospital from January 2016 to December 2020 were selected as the study group, and the children with Influenza-Associated Encephalopathy (IAE) group who received treatment in the same period were selected as the control group. The blood biochemical, coagulation function and cerebrospinal fluid test results of the two groups were analyzed by univariate analysis. Receiver Operating Characteristic Curve (ROC) analysis was used to determine the optimal threshold point of each index for the indicators with statistically significant differences in univariate analysis results, and multivariate Logistic stepwise regression analysis was performed according to the optimal threshold points.

Results: In the IANE group, there were 32 children, including 20 males and 12 females, aged $58(23,97)$ months. There were 40 children in IAE group, including 26 males and 14 females, aged $58(23,97)$ months. Univariate results showed that serum Lactate Dehydrogenase (LDH), Cerebrospinal Fluid Lactate Dehydrogenase (CSF LDH) and Cerebrospinal Fluid Protein (CSF Pro) in the IANE group were significantly higher than those in the IAE group, and the difference between the two groups was statistically significant $(P<0.001)$. The optimal threshold points of blood LDH, CSF LDH and CSF Pro by ROC curve analysis were $535 \mathrm{U} / \mathrm{L}, 67 \mathrm{U} / \mathrm{L}$ and $0.49 \mathrm{~g} / \mathrm{L}$, respectively. Further Multivariate Logistic stepwise regression analysis showed that $\mathrm{LDH}>535 \mathrm{U} / \mathrm{L}(\mathrm{OR}=31.264$, 95\% Cl: $5.892-165.878, \mathrm{P}<0.001)$ and CSF PRO $>0.49 \mathrm{~g} / \mathrm{L}(\mathrm{OR}=7.695,95 \% \mathrm{Cl}$ : 1.052-56.305, $\mathrm{P}=0.044$ ) were independent risk factors for IANE.

Conclusion: For children with influenza whose neurological symptoms appear rapidly and persist in the early stages of the disease, blood $\mathrm{LDH}>535 \mathrm{U} / \mathrm{L}$ and CSF PRO $>0.49 \mathrm{~g} / \mathrm{L}$ are independent risk factors for IANE.

Keywords: Children; Influenza-associated necrotizing encephalopathy; Risk factor

\section{Introduction}

Influenza occurs frequently in winter and spring in southern China. Most infected children have fever and respiratory symptoms. In addition to fever and respiratory symptoms, some children with influenza will have neurological symptoms, manifested as convulsions, disturbance of consciousness and coma. Some cases will die after rapid progress, and the survivors will have severe neurological sequelae [1-3]. Nervous system damage caused by influenza is one of the main causes of influenza death in children, among which IANE is the most serious, with a case fatality rate of up to $30 \%[4,5]$. However, there is currently a lack of indicators related to IANE risk factors, which delays the early identification of critical cases, leading to poor prognosis and death [6].

This study retrospectively analyzed the blood biochemical, coagulation function and cerebrospinal fluid results of children with IAE and IANE who were admitted to our hospital from January
2016 to December 2020 with neurological symptoms, to explore the possibility of predicting IANE in the early stage of the disease, so as to detect the children with IANE early, and to closely evaluate and actively intervene in these children to improve the prognosis. Reduce the nervous system sequelae and mortality.

\section{Patients and Methods}

\section{Clinical data}

The gender, age, blood biochemical results, blood coagulation function, cerebrospinal fluid results, Imaging data and prognosis of children with IAE and IANE treated in Guangzhou Women and Children's Medical Center from January 2016 to December 2020 were retrospectively analyzed. The Ethics Committee of Guangzhou Women and Children Medical Center approved this study. All patients signed an informed consent form upon admission.

The inclusion criteria were: 1$)$ children (<18 years); 2$)$ admitted to the hospital with influenza virus infection; and 3) neurological 
manifestations during hospitalization. The exclusion criteria were: 1) admission $>7$ days after onset; 2) co-infected with other pathogens; 3) comorbidities like brain trauma, sequelae of viral encephalitis, or metabolic diseases; 4) missing data; or 5) neurological complications other than IAE or IANE.

IAE was defined as convulsions, acute cognitive impairment, and acute disturbance of consciousness, and coma [7-9]; without specific biochemistry abnormal, without or minor imaging changes such as slight cerebral edema [10]. IANE was defined as acute fever, frequent convulsions, acute disturbance of consciousness, coma, and multiple organ failure, with a risk of death [11-13]; biochemistry changes could be not specific [10], but imaging shows brain edema and necrosis of thalamus and other deep brain structures $[10,14]$.

\section{Methods}

Through the data query of structured electronic medical record system, the gender, age, blood biochemical results and cerebrospinal fluid results of the first test after hospitalization, imaging data and prognosis of children diagnosed with IAE and IANE admitted to our hospital from January 2016 to December 2020 were analyzed by retrospective analysis. The collected clinical data and laboratory examination results were statistically analyzed.

\section{Statistical analysis}

All data were processed using SPSS (V26.0, IBM Corp, Armonk, NY, USA) statistical software. Enumerative data were expressed by numerical values and percentages. Differences between groups were tested by Pearson chi-square $\left(\chi^{2}\right)$ test or Fisher's exact test. ShapiroWilk method was used to test the normality of measurement data, and the interval between median and quaternary M (P25, P75) was used to represent the non-normal distribution, and the differences between groups were tested by Mann-Whitney U test. Mean \pm SD was used for normal distribution, and $t$ test was used for comparison between groups. $\mathrm{P}$ value $<0.05$ on both sides was considered statistically significant. ROC curve analysis was used to calculate the area under the curve and determine the optimal threshold value. Multivariate logistic stepwise regression analysis was performed on the variables with statistically significant differences in univariate analysis.

\section{Results}

\section{Characteristics of cases}

From January 2016 to December 2020, a total of 72 children with influenza associated with neurological complications were admitted to our hospital. In the IANE group, there were 32 children, including 20 males and 12 females, aged $58(23,97)$ months, and all of them had an initial infection and persistent neurological symptoms following, such as Acute Disturbance of Consciousness (ADOC) or seizure occurred within three days after onset of the disease. There were 40 children in IAE group, including 26 males and 14 females, aged $58(23,97)$ months, whose neurological symptoms were milder and shorter than IANE group. There was no significant difference in gender ratio and age distribution between the two groups $(\mathrm{P}>0.05)$ (Table 1).

\section{Imaging data}

Among the 40 children with IAE, 37 (92.5\%) underwent brain Computerized Tomography (CT) or Magnetic Resonance Imaging
Table 1: Analysis of patient data and laboratory results of the two groups.

\begin{tabular}{|c|c|c|c|c|}
\hline Variable & IAE $(n=40)$ & IANE $(n=32)$ & $X^{2} / t / U$ & $\mathbf{P}^{*}$ \\
\hline Male/Female & $26 / 14$ & $20 / 12$ & 0.048 & 0.826 \\
\hline Age/Month & $58(23,97)$ & $60(35,84)$ & 437 & 0.545 \\
\hline \multicolumn{5}{|l|}{ Blood biochemical } \\
\hline AST/U.L-1 & $37(33,47)$ & $49(35,101)$ & 815 & 0.026 \\
\hline ALT/U.L-1 & $18(12,20)$ & $25(14,48)$ & 776.5 & 0.013 \\
\hline CK/U.L $L^{-1}$ & $174(115,279)$ & $239(159,356)$ & 440 & 0.036 \\
\hline CK-MB/U.L-1 & $15(9,26)$ & $19(12,33)$ & 347 & 0.105 \\
\hline Cr/umol..L-1 & $32(24,38)$ & $41(23,49)$ & 1842 & 0.689 \\
\hline Urea/mmol.L-1 & $3.3(93.06,4.40)$ & $3.78(3.10,5.30)$ & 655 & 0.164 \\
\hline $\mathrm{LDH} / \mathrm{U} . \mathrm{L}^{-1}$ & $336(218,493)$ & $567(369,916)$ & 109.5 & $<0.001$ \\
\hline \multicolumn{5}{|l|}{ Coagulation } \\
\hline PT/S & $14.66 \pm 1.08$ & $16.79 \pm 5.21$ & 1.514 & 0.153 \\
\hline TT/S & $17.47 \pm 1.76$ & $19.58 \pm 4.29$ & 1.773 & 0.096 \\
\hline APTT/S & $45.97 \pm 14.39$ & $49.45 \pm 10.82$ & 0.651 & 0.522 \\
\hline $\mathrm{FIB} / \mathrm{g} \cdot \mathrm{L}^{-1}$ & $3.05 \pm 0.88$ & $2.63 \pm 0.92$ & 1.444 & 0.156 \\
\hline \multicolumn{5}{|l|}{ Cerebrospinal fluid } \\
\hline CSF leukocyte $/ 10^{6} \cdot L^{-1}$ & $3(1,4)$ & $4(1,6)$ & 326 & 0.094 \\
\hline CSF GLU/mmol.L-1 & $3.44(3.20,3.67)$ & $3.71(3.17,4.27)$ & 356 & 0.231 \\
\hline CSF LDH/U.L-1 & $21(16,24)$ & $76(24,145)$ & 127.5 & $<0.001$ \\
\hline CSF PRO/g. $\mathrm{L}^{-1}$ & $0.28(0.21,0.39)$ & $0.65(0.43,0.98)$ & 147 & $<0.001$ \\
\hline \multicolumn{5}{|l|}{ The prognosis } \\
\hline Sequelae (\%) & $14(35)$ & $17(53)$ & 2.382 & 0.123 \\
\hline Died (\%) & $0(0)$ & $10(31)$ & 12.021 & 0.001 \\
\hline
\end{tabular}

Note: IAE: Influenza-Associated Encephalopathy; IANE: Influenza-Associated Necrotizing Encephalopathy; AST: Aspartate Aminotransferase; Alt: Alanine Aminotransferase; CK: Creatinine Kinase; CK-MB: Creatine Kinase Isoenzyme; Cr: Creatinine; Urea Nitrogen; LDH: Lactate Dehydrogenase; Pt: Prothrombin Time; TT: Thrombin Time; APTT: Activated Partial Thromboplastin Time; Fib: Fibrinogen; CSF Leukocyte: CSF Leukocyte; CSF Glu: Cerebrospinal Fluid Glucose; CSF LDH: Cerebrospinal Fluid Lactate Dehydrogenase; CSF Pro: Cerebrospinal Fluid Protein; *Measurement data, Mann-Whitney $U$ test or $t$ test; Enumeration data, $\mathrm{X}^{2}$ test or Fisher's exact test.

(MRI) examination, and $15(40.5 \%)$ of them showed abnormal manifestations, slight cerebral edema mainly. Twenty-eight of the $32(87.5 \%)$ children with IANE underwent brain CT or MRI examination, and all of them (100\%) showed abnormal findings, mainly symmetrical lesions in the thalamus and other brain structures, especially in the brainstem, basal ganglia, periventricular white matter and cerebellum. Brain MRI findings of an IANE patient was showed in the Figure 1.

\section{Laboratory results and prognosis}

The blood biochemical results, coagulation function, Cerebrospinal Fluid (CSF) results and prognosis of 40 IAE patients and 32 IANE patients were statistically analyzed (Table 1). In terms of blood biochemical results, Aspartate Aminotransferase (AST) of IAE group and IANE group were $37(33,47) \mathrm{U} / \mathrm{L}$ and $49(35,101) \mathrm{U} / \mathrm{L}$, Alanine Aminotransferase (ALT) were $18(12,20) \mathrm{U} / \mathrm{L}$ and $25(14,48)$ $\mathrm{U} / \mathrm{L}$, creatinine kinase $(\mathrm{CK})$ were $174(115,279) \mathrm{U} / \mathrm{L}$ and $239(159,356)$ $\mathrm{U} / \mathrm{L}$, Creatine Kinase Isoenzyme (CK-MB) were $15(9,26) \mathrm{U} / \mathrm{L}$ and 19 $(12,33) \mathrm{U} / \mathrm{L}$, Creatinine $(\mathrm{Cr})$ were $32(24,38) \mathrm{Umol} / \mathrm{L}$ and $41(23,49)$ 


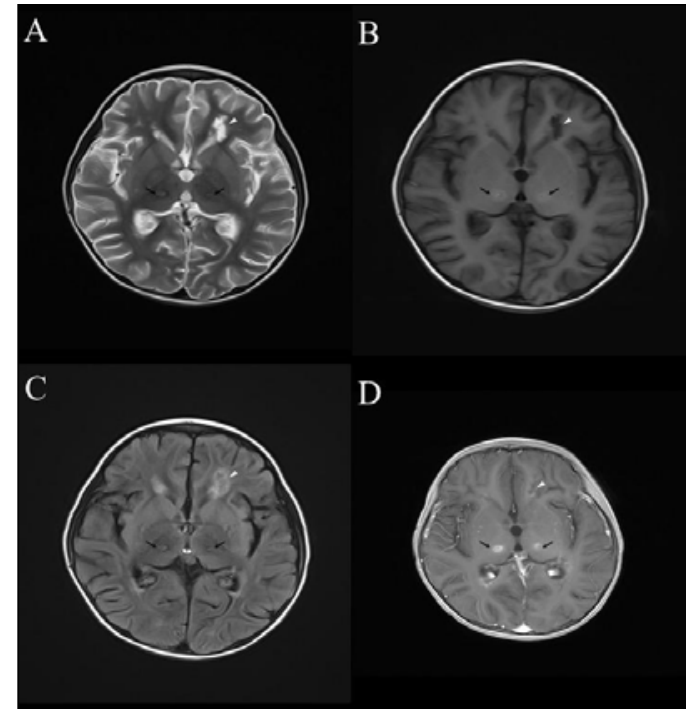

Figure 1: Brain MRI findings of a child in the IANE group.

Note: A) Symmetrical multilayer concentric circles of bilateral thalamic lesions (black arrows) on axial T2WI show slightly higher signal in the center, slightly lower signal in the middle, and patch like slightly higher signal in the periphery with blurred edges. Hyperintensity is seen in the left ventricular voiceover lesion (white arrow). B) Axial T1WI bilateral thalamic lesions (black arrow) show slightly higher signal in the center, slightly lower signal in the middle, and patch like slightly higher signal in the periphery with blurred edges. The voiceover lesion (white arrow) of the left ventricle shows low signal. C) Axial T2-FLAIR bilateral thalamic lesions (black arrow) showed slightly higher signal in the center, slightly higher signal in the center, slightly lower signal in the middle, and patch like slightly higher signal in the peripheral part. Slight hyperintensity in the left ventricular voiceover lesion (white arrow); D) Axial enhancement Bilateral thalamic lesions (black arrows) on T1WI show no enhancement in the center and periphery, and ring enhancement in the middle. Small patches of enhancement are seen at the margin of the peripheral mass of the left ventricle (white arrow).

umol /L, Urea were $3.39(3.06,4.40) \mathrm{mmol} / \mathrm{L}$ and $3.78(3.10,5.30)$ $\mathrm{mmol} / \mathrm{L}, \mathrm{LDH}$ were $336(218,493) \mathrm{U} / \mathrm{L}$ and $567(369,916) \mathrm{U} / \mathrm{L}$. In terms of blood coagulation function, Prothrombin Time (PT) of IAE group and IANE group were $14.66 \pm 1.08 \mathrm{~s}$ and $16.79 \pm 5.21 \mathrm{~s}$, Thrombin Time (TT) were $17.47 \pm 1.76 \mathrm{~s}$ and $19.58 \pm 4.29 \mathrm{~s}$, Activated Partial Thromboplastin Time (APTT) were $45.97 \pm 14.39 \mathrm{~s}$ and $49.45 \pm 10.82 \mathrm{~s}$, fibrinogen (FIB) was $3.05 \pm 0.88 \mathrm{~g} / \mathrm{L}$ and $2.63 \pm 0.92 \mathrm{~g} / \mathrm{L}$. In terms of CSF results, CSF leukocyte levels in IAE group and IANE group were 3 $(1,4){ }^{\star} 10^{\wedge} 6 / \mathrm{L}$ and $4(1,6){ }^{\star} 10^{\wedge} 6 / \mathrm{L}$, cerebrospinal fluid glucose (CSF Glu) were $3.44(3.20,3.67) \mathrm{mmol} / \mathrm{L}$ and $3.71(3.17,4.27) \mathrm{mmol} / \mathrm{L}$, CSF LDH was $21(16,24) \mathrm{U} / \mathrm{L}$ and $76(24,145) \mathrm{U} / \mathrm{L}$, CSF Pro was $0.28(0.21,0.39) \mathrm{g} / \mathrm{L}$ and $0.65(0.43,0.98) \mathrm{g} / \mathrm{L}$. In terms of prognosis, the sequelae of IAE and IANE group were $14(35 \%)$ and $15(53 \%)$, death were $0(0 \%)$ and 10 (mortality rate was $31 \%$ ).

The results showed that there were no statistical differences in CK-MB, Cr, Urea, PT, TT, APTT, FIB, CSF leukocyte, CSF Glu and sequelae between the two groups $(\mathrm{P}>0.05)$. Comparing with IAE group, the differences of AST, ALT and CK in IANE group were statistical $(\mathrm{P}<0.05)$, and that in IANE group was higher than that in IAE group. Blood LDH, CSF LDH and CSF Pro had statistically significant differences $(\mathrm{P}<0.001)$, and the IANE group was significantly higher than the IAE group. There was no statistically difference in sequelae between the two groups $(\mathrm{P}>0.05)$, and the difference in mortality was

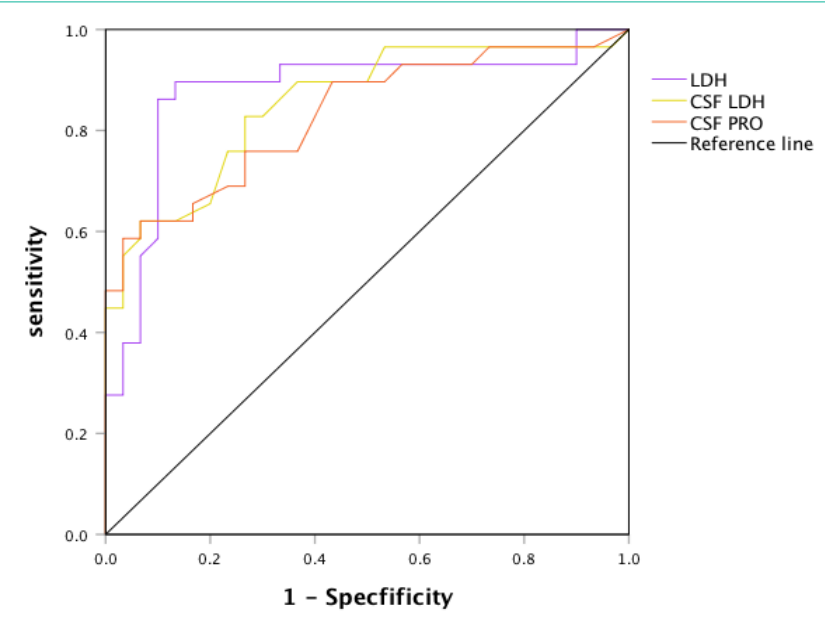

Figure 2: The ROC curve of LDH, CSF LDH and CSF Pro to predict IANE.

Table 2: Efficiency of predicting IANE by various indicators.

\begin{tabular}{|l|c|c|c|c|}
\hline \multicolumn{1}{|c|}{ Laboratory } & AUC & Sensitivity & Specificity & Cutoff \\
\hline LDH & 0.876 & 89.7 & 86.5 & 535 \\
\hline CSF LDH & 0.853 & 82.8 & 73.3 & 67 \\
\hline CSF PRO & 0.831 & 73.7 & 76.6 & 0.49 \\
\hline
\end{tabular}

Note: IANE: Influenza-Associated Necrotizing Encephalopathy; LDH: Lactate Dehydrogenase; CSF LDH: Cerebrospinal Fluid Lactate Dehydrogenase; CSF Pro: Cerebrospinal Fluid Protein.

Table 3: Risk factors for IANE by multivariate analysis.

\begin{tabular}{|l|c|c|c|c|c|c|}
\hline \multirow{2}{*}{ Variables } & \multirow{2}{*}{$\boldsymbol{\beta}$} & SE & P & OR & \multicolumn{2}{c|}{$95 \% \mathrm{Cl}$} \\
\cline { 6 - 8 } & & & & & Lower & Upper \\
\hline LDH $>535 \mathrm{U} / \mathrm{L}$ & 3.442 & 0.851 & $<0.001$ & 31.264 & 5.892 & 165.878 \\
\hline CSF PRO $>0.49 \mathrm{~g} / \mathrm{L}$ & 2.041 & 1.015 & 0.044 & 17.695 & 1.052 & 56.305 \\
\hline Constant & -8.593 & 2.157 & $<0.001$ & & & \\
\hline
\end{tabular}

Note: LDH: Lactate Dehydrogenase; CSF Pro: Cerebrospinal Fluid Protein.

statistical difference $(\mathrm{P}<0.05)$.

\section{ROC curve analysis}

Since LDH, CSF LDH and CSF Pro of the IANE group were significantly higher than those of the IAE group were; ROC curve analysis was performed on the three indicators to determine the area under the curve, the optimal threshold point, sensitivity and specificity of each indicator (Figure 2). The Area under Curve (AUC) of blood LDH, CSF LDH and CSF Pro were 0.876, 0.853 and 0.831, respectively (Table 2). The optimal threshold points of blood $\mathrm{LDH}$, CFS LDH and CSF Pro were 535U/L, 67U/L and 0.49g/L, respectively. The sensitivity and specificity of LDH $>535 \mathrm{U} / \mathrm{L}$ for predicting IANE were $89.7 \%$ and $86.5 \%$ respectively. The sensitivity and specificity of $\mathrm{CSF} \mathrm{LDH}>67 \mathrm{U} / \mathrm{L}$ for predicting IANE were $82.8 \%$ and $73.3 \%$ respectively. The sensitivity and specificity of CSF Pro $>0.49 \mathrm{~g} / \mathrm{L}$ for predicting IANE was $73.7 \%$ and $76.6 \%$ respectively.

\section{Logistic regression analysis}

Logistic stepwise regression analysis was conducted on the three variables with statistically significant difference results from univariate analysis according to the optimal threshold point determined by ROC analysis; two variables were included in the final regression model (Table 3). The analysis results showed that the OR value of blood 
LDH $>535 \mathrm{U} / \mathrm{L}$ was $31.264,95 \%$ CI: $5.892-165.878, \mathrm{P}<0.001$. The OR value of CSF Pro $>0.49 \mathrm{~g} / \mathrm{L}$ was $7.695,95 \%$ CI: $1.052-56.305, \mathrm{P}=0.044$. Both of the two variables were independent risk factors for IANE.

\section{Discussion}

Influenza has different characteristics of prevalence and high incidence in different regions. Temperate regions have high prevalence in winter and spring, while tropical regions show diversified seasonality, with either semi-annual or year-round periodic epidemics [15-17]. In the influenza epidemic season, the infection rate of children can reach 20\% 30\%, and even in the high epidemic season, the annual infection rate can reach about 50\% $[18,19]$. Neurological system damage is one of the serious complications of influenza. An epidemiological survey in Japan showed that the proportion of nervous system damage in children under 18 years old accounted for $74 \%$ and the case fatality rate was $9 \%$ [20]. As the same as ANE causing by a variety of causes [21], the mortality rate of IANE is about 30\% [9], which was close to our findings (Table 1).

Flu infections ANE progress rapidly, sequela and high mortality rate, early diagnosis and intervention is the key. The current IANE diagnosis basis for the clinical manifestation and the evaluation of the nervous system examination, IANE brain imaging studies including CT and MRI. As other studies showed, our study found the symmetry of thalamus and other brain structures lesions, particularly in the brainstem, basal ganglia, white matter around ventricle and cerebellum in IANE group. However, due to the limitations of illness, examination conditions and caregivers' wishes, neuroimaging examination cannot be carried out in all children, and simple and feasible risk factors related indicators for the occurrence of IANE are needed clinically.

In this study, the data of 40 cases of IAE and 32 cases of IANE who were admitted to our hospital from January 2016 to December 2020 with influenza infection and nervous system symptoms were retrospective analyzed. The results showed that there were statistically differences in ALT, AST and CK between the two groups $(\mathrm{P}<0.05)$, and the IANE group was higher than the IAE group. This is consistent with previous literature reports that children with IANE have varying degrees of elevated AST, ALT and CK, which may be related to the fact that children with IANE are more prone to liver function and muscle injury [22,23]. There were statistically significant differences in serum LDH, CSF LDH and CSF Pro between the two groups (P $<0.001$ ), and the IANE group was significantly higher than the IAE group. $\mathrm{LDH}$ in serum and cerebrospinal fluid of the IANE group was significantly higher than that of the IAE group, the reason being that LDH could be overexpressed in cell necrosis [24-26]. The assessment of LDH in serum and cerebrospinal fluid is helpful for the assessment of peripheral and brain cell necrosis. The increase of CSF protein in the IANE group was associated with brain cell necrosis and injury, which was consistent with previous literature that CSF protein in the IANE group was greater than $0.4 \mathrm{~g}[13,27]$.

ROC curve analysis of serum LDH, CSF LDH and CSF Pro showed that the areas under the curve of LDH, CSF LDH and CSF Pro were $0.876,0.853$ and 0.831 , respectively. The optimal threshold points of LDH, CSF LDH and CSF Pro were 535U/L, 67U/L and $0.49 \mathrm{~g} / \mathrm{L}$, respectively. The sensitivity and specificity of blood $\mathrm{LDH}$
$>535 \mathrm{U} / \mathrm{L}$, CSF LDH $>67 \mathrm{U} / \mathrm{L}$, CSF Pro $>0.49 \mathrm{~g} / \mathrm{L}$ were $89.7 \%, 82.8 \%$, $73.7 \%$ and $86.5 \%, 73.3 \%, 76.6 \%$, respectively. All three indexes had good sensitivity and specificity in predicting IANE. Logistic stepwise regression analysis was conducted for the three indicators according to the optimal threshold points determined by ROC analysis, and the results showed that two variables were included in the final regression model. The analysis results indicated that $\mathrm{LDH}>535 \mathrm{U} / \mathrm{L}$ predicted the risk of IANE 31.264 times as much as $\mathrm{LDH} \leq 535 \mathrm{U} / \mathrm{L}$ ( $\mathrm{OR}=31.264$, 95\% CI: 5.892-165.878), CSF Pro $>0.49 \mathrm{~g} / \mathrm{L}$ predicted the risk of IANE 7.695 times as much as CSF Pro $\leq 0.49 \mathrm{~g} / \mathrm{L}(\mathrm{OR}=7.695,95 \%$ CI: 1.052 56.305), both of which are independent risk factors for IANE and have high predictive value for IANE.

\section{Conclusion}

For children with influenza whose neurological symptoms appear rapidly and persist in the early stages of the disease, blood $\mathrm{LDH}>535 \mathrm{U} / \mathrm{L}$ and CSF $\mathrm{PRO}>0.49 \mathrm{~g} / \mathrm{L}$ are independent risk factors for IANE, that need high vigilance, close evaluation and active intervention should be taken to reduce sequelae and mortality and improve the prognosis.

\section{References}

1. Mizuguchi M, Ichiyama T, Imataka G, Okumura A, Goto T, Sakuma H, et al. Guidelines for the diagnosis and treatment of acute encephalopathy in childhood. Brain \& development. 2021; 43: 2-31.

2. Welk A, Schmeh I, Knuf M, Groendahl B, Goebel J, Staatz G, et al. Acute Encephalopathy in Children Associated with Influenza A: A Retrospective Case Series. Klin Padiatr. 2016; 228: 280-281.

3. Chen LW, Teng CK, Tsai YS, Wang JN, Tu YF, Shen CF, et al. Influenzaassociated neurological complications during 2014-2017 in Taiwan. Brain \& development. 2018; 40: 799-806.

4. Akins PT, Belko J, Uyeki TM, Axelrod Y, Lee KK, Silverthorn J. H1N1 encephalitis with malignant edema and review of neurologic complications from influenza. Neurocrit Care. 2010; 13: 396-406.

5. Britton PN, Dale RC, Blyth CC, Macartney K, Crawford NW, Marshall H, et al. Influenza-associated Encephalitis/Encephalopathy Identified by the Australian Childhood Encephalitis Study 2013-2015. The Pediatric infectious disease journal. 2017; 36: 1021-1026.

6. Ekstrand JJ. Neurologic complications of influenza. Semin Pediatr Neurol. 2012; 19: 96-100.

7. Sugaya N. Influenza-associated encephalopathy in Japan. Seminars in pediatric infectious diseases. 2002; 13: 79-84.

8. Shiomi M: [Pathogenesis of acute encephalitis and acute encephalopathy]. Nihon rinsho Japanese journal of clinical medicine. 2011; 69: 399-408.

9. Togashi T, Matsuzono Y, Narita M, Morishima T. Influenza-associated acute encephalopathy in Japanese children in 1994-2002. Virus research. 2004; 103: 75-78.

10. Chen Q, Li P, Li S, Xiao W, Yang S, Lu H. Brain Complications with Influenza Infection in Children. Journal of Behavioral and Brain Science. 2020; 10: 129152.

11. Howard A, Uyeki TM, Fergie J. Influenza-Associated Acute Necrotizing Encephalopathy in Siblings. Journal of the Pediatric Infectious Diseases Society. 2018; 7: e172-e177.

12. Weitkamp JH, Spring MD, Brogan T, Moses H, Bloch KC, Wright PF. Influenza A virus-associated acute necrotizing encephalopathy in the United States. The Pediatric infectious disease journal. 2004; 23: 259-263.

13. Mizuguchi M, Abe J, Mikkaichi K, Noma S, Yoshida K, Yamanaka T, et al. Acute necrotising encephalopathy of childhood: a new syndrome presenting with multifocal, symmetric brain lesions. Journal of neurology, neurosurgery, and psychiatry. 1995; 58: 555-561. 
14. Kirat N, De Cauwer H, Ceulemans B, Vanneste D, Rossi A. Influenzaassociated encephalopathy with extensive reversible restricted diffusion within the white matter. Acta neurologica Belgica. 2018; 118: 553-555.

15. Azziz Baumgartner E, Dao CN, Nasreen S, Bhuiyan MU, Mah EMS, A Mamun A, et al. Seasonality, timing, and climate drivers of influenza activity worldwide. The Journal of infectious diseases. 2012; 206: 838-846.

16. Bloom-Feshbach K, Alonso WJ, Charu V, Tamerius J, Simonsen L, Miller MA et al. Latitudinal variations in seasonal activity of influenza and respiratory syncytial virus (RSV): a global comparative review. PLoS One. 2013; 8 : e54445.

17. Zou J, Yang H, Cui H, Shu Y, Xu P, Xu C, et al. Geographic divisions and modeling of virological data on seasonal influenza in the Chinese mainland during the 2006-2009 monitoring years. PLoS One. 2013; 8: e58434.

18. Fraaij PL, Heikkinen T. Seasonal influenza: the burden of disease in children. Vaccine. 2011; 29: 7524-7528.

19. Cowling BJ, Perera RA, Fang VJ, Chan KH, Wai W, So HC, et al. Incidence of influenza virus infections in children in Hong Kong in a 3-year randomized placebo-controlled vaccine study, 2009-2012. Clinical infectious diseases: an official publication of the Infectious Diseases Society of America. 2014; 59 : 517-524.

20. Okuno H, Yahata Y, Tanaka-Taya K, Arai S, Satoh H, Morino S, et al Characteristics and Outcomes of Influenza-Associated Encephalopathy Cases Among Children and Adults in Japan, 2010-2015. Clinical infectious diseases: an official publication of the Infectious Diseases Society of America. 2018; 66: 1831-1837.
21. Wu X, Wu W, Pan W, Wu L, Liu K, Zhang HL. Acute necrotizing encephalopathy: an under recognized clinicoradiologic disorder. Mediators Inflamm. 2015; 2015: 792578.

22. Lee YJ, Hwang SK, Kwon S. Acute Necrotizing Encephalopathy in Children: a Long Way to Go. J Korean Med Sci. 2019; 34: e143.

23. Trujillo-Gomez J, Cabrera-Hemer DN: [Acute necrotizing encephalopathy]. Rev Neurol. 2019; 69: 349-350.

24. Huang CF, Liu SH, Lin-Shiau SY. Pyrrolidine dithiocarbamate augments $\mathrm{Hg}(2+)$-mediated induction of macrophage cell death via oxidative stressinduced apoptosis and necrosis signaling pathways. Toxicol Lett. 2012; 214 33-45.

25. Franke RP, Fuhrmann R, Mrowietz C, Rickert D, Hiebl B, Jung F. Reduced diagnostic value of lactate dehydrogenase (LDH) in the presence of radiographic contrast media. Clin Hemorheol Microcirc. 2010; 45: 123-130.

26. Tran TT, Groben P, Pisetsky DS. The release of DNA into the plasma of mice following hepatic cell death by apoptosis and necrosis. Biomarkers. 2008; 13: $184-200$.

27. Wang HS. Acute necrotising encephalopathy of childhood presenting with multifocal, symmetric brain lesions occurring outside Japan. Journal of neurology, neurosurgery, and psychiatry. 1995; 59: 661. 